Pacific Journal of Mathematics

PREDICTION THEORY FOR MARKOFF PROCESSES 


\section{PREDICTION THEORY FOR MARKOFF PROCESSES}

\section{A. V. BALAKRISHNAN}

In this paper we consider the least square prediction problem for Markoff processes with stationary transitions. The main result concerns the partial differential equation characterizing the prediction operator, and the conditions for the uniqueness of the solutions.

Introduction. Let $x(t)$ be a Markoff process with stationary transitions. It is well-known that the optimum mean square predictor of $g(x(s+t))$ given $x(\sigma)$ for $\sigma \leqq s$ is given by the conditional expectation:

$$
E[g(x(t+s)) \mid x(\sigma) \leqq s] .
$$

For a Markoff process this becomes

$$
E\left[g(x(t+s)) \Gamma_{.} x(s)\right]
$$

and further, if the transitions are stationary, we need only to consider:

$$
E[g(x(t)) \mid x(0)]
$$

Let $p(t, \xi \mid x)$ be the distribution function (suitably normalized) of the conditional or transition probability of transition from $x$ to $\xi$ in time $t$. Then, of course, (1.2) becomes

$$
\int g(\xi) d_{\xi} p(t, \xi \mid x) .
$$

Now if $g($.$) is in C[\alpha, \beta]$, where $-\infty \leq \alpha<\beta \leq+\infty$ is the interval over which the transition probabilities are defined, we obtain a semigroup of linear operators over $C[\alpha, \beta]$ defined through (1.3). If now we know the infinitesimal generator of this semigroup, we obtain an abstract differential equation for (1.3):

$$
\frac{d u(t, g)}{d t}=\Lambda u(t, g)
$$

where $u(t, \mathrm{~g})$ represents (1.3) and $A$ is the infinitesimal generator, provided $g($.$) is in the domain of \Lambda$. If we know the representation of $A$, and if in particular, it turns out to be a partial differential operator, (1.4) offers an alternate way of determing the prediction functions (1.2) provided uniqueness of the solution can be proved. In what follows, we shall be concerned primarily with situations where such a reduction is possible, and the associated conditions for uniqueness.

Received September 1960. 
Main Results:

2. Markoff processes of the diffusion type. A well-known set of sufficient conditions under which the reduction to a parabolic partial differential equation is possible are the Lindberg-Levy conditions which we state here in their weakest form due to Feller $[3,4]$. Let

$$
\begin{aligned}
& \text { (i) } \frac{1}{t} \int_{|\xi-x|>\varepsilon} d_{\xi} P(t, \xi \mid x) \rightarrow 0 \text { as } t \rightarrow 0+ \\
& L_{1} \quad \text { (ii) } \frac{1}{t} \int_{|\xi-x|<\varepsilon}(\xi-x) d_{\xi} P(t, \xi \mid x) \rightarrow b(x) \text { as } t \rightarrow 0+ \\
& \quad \text { (iii) } \frac{1}{t} \int_{|\xi-x|<\varepsilon}(\xi-x)^{2} d_{\xi} P(t, \xi \mid x) \rightarrow 2 a(x) \text { as } t \rightarrow 0+
\end{aligned}
$$

Then for each $g($.$) in C[\alpha, \beta]$, if we set:

$$
T(t) g(x)=\int_{\alpha}^{\beta} g(\xi) d_{\xi} P(t, \xi \mid x) .
$$

$T(t)$ is a semigroup of linear bounded operators over $[\alpha, \beta]$ and moreover

(i ) $\|T(t) g\| g \|$ (contraction semigroup)

(ii) $\|T(t) g-g\| \rightarrow 0$ as $t \rightarrow 0+$ (strongly continuous)

(iii) $T(t) g$ is non-negative if $g$ is nonnegative. (positivity preserving)

(vi) For $g(\xi)=$ constant $T(t) g=g$.

Properties (i), (iii) and (iv) are obvious from (2.1). That $T(t) g$ again belongs to $C[\alpha, \beta]$ follows from condition (i) of $L_{1}$, and so does property (ii). Let $\Lambda$ be the infinitesimal generator of the semigroup. Then the most important property one would like to deduce from $L_{1}$ is that it coincides with a second-order differential operator. Unfortunately, however, this is not always entirely true. For example following Feller [4], suppose we define the transition density kernels,

$$
P(t, \xi \mid x)=\frac{1}{\sqrt{2 \pi t}}\left[\exp -\frac{(g(\xi)-g(x))^{2}}{2 t}\right] g^{\prime}(\xi), \alpha=-\infty, \beta=+\infty
$$

where say $g($.$) is a polynomial which vanishes at the origin, and g^{\prime}(\xi)>0$. Taking $g(\xi)=\xi^{3}$, we obtain for $\xi \neq 0$

$$
\begin{aligned}
& b(\xi)=-\frac{2}{9} \xi^{-5} \\
& a(\xi)=\frac{\xi^{-4}}{9} .
\end{aligned}
$$

However, at $\xi=0$, 


$$
\begin{aligned}
& a(0)=0 \\
& b(0)=0 .
\end{aligned}
$$

Direct substitution into (2.1) shows that for $f($.$) in the domain of \Lambda$, $f(0)=f^{\prime}(0)=f^{\prime \prime}(0)$ and that

$$
\Lambda f(0)=\frac{2}{6 !} f^{6}(0) \text {. }
$$

Although for any $\xi \pm 0$,

$$
\Lambda f(\xi)=a(\xi) \frac{d^{2} f}{d \xi^{2}}+b(\xi) \frac{d f}{d \xi} .
$$

Here it may be noted that the exceptional point zero is a point of discontinuity of the functions $a($.$) and b($.$) . One might then expect$ that this may avoided if they are required to be continuous. However, it should be noted even in this case that $\Lambda$ may not still coincide entirely with the differential operator on the right in (2.2)-in fact, it may only be a contraction of that operator. With some additional conditions on $a(\xi)$ and $b(\xi)$ we can nevertheless obtain a stronger result.

Theorem 2.1. Let $a(\xi), b(\xi)$ given by $L_{1}$ be continuously twice differentiable in the open interval $(\alpha, \beta)$ and $a(\xi)>0$ therein. Let the limits in $L_{1}$ hold uniformly in $x$ in each compact sub-interval. Suppose in addition they satisfy:

$$
\int_{0}^{\infty} q(\xi) w(\xi) d \xi=+\infty=\int_{0}^{\beta} q(\xi) w(\xi) d \xi
$$

where $\alpha<0<\beta$

$$
\begin{aligned}
& q(t)=\int_{0}^{t} \frac{d t}{a(t) w(t)} \\
& w(t)=\exp -\int_{0}^{t} \frac{b(\xi)}{a(\xi)} d \xi .
\end{aligned}
$$

Then the infinitesimal generator $\Lambda$ of the semigroup coincides with the differential operator $C$

$$
C=a(\xi) \frac{d^{2}}{d \xi^{2}}+b(\xi) \frac{d}{d \xi}
$$

where the domain of $C$ consists of functions $f(\xi)$ with first and second derivatives such that

$$
a(\xi) \frac{d^{2} f}{d \xi^{2}}+b(\xi) \frac{d f(\xi)}{d \xi}
$$


belongs to $C[\alpha, \beta]$.

Conversely suppose the functions $a(\xi)$ and $b(\xi)$ are given, with $a(\xi)$ positive and continuous and $b(\xi)$ continuous in the open interval $[\alpha, \beta]$, and suppose (2.3) is satisfied. Then $C$ generates a semigroup given by (2.1) where the kernels are Markoff transition probabilities which satisfy the conditions $L_{1}$, the limits holding uniformly in $x$ in each compact. sub-interval.

Proof. Let us consider the converse statement first. Under the conditions (2.3) on the coefficients $a(\xi)$ and $b(\xi)$, Hille [5] has shown that $C$ is the infinitesimal generator of a strongly continuous positive contraction semigroup. Denoting this semigroup by $S(t)$, we have, for any $f($.$) in C[\alpha, \beta]$ :

$$
S(t) f(x)=\int_{\alpha}^{\beta} f(\xi) d_{\xi} P(t, \xi ; x)
$$

where the $P(t, \xi ; x)$ are Markoff transition kernels. Moreover, it is readily shown that the kernels satisfy the conditions $L_{1}$, with the necessary uniformity.

Suppose next that we are given transition probabilities satisfying $L_{1}$ where $a($.$) and b($.$) satisfy (2.3). We know then (2.1) yields a strongly$ continuous semigroup, and we have to show that its infinitesimal generator $A$ coincides with $C$. For this, suppose $f($.$) is in the domain of C^{2}$. Then $f($.$) has first and second derivatives. Further, suppose f^{\prime}($.$) vani-$ shes outside a compact sub-interval, say $\left[r_{1}, r_{2}\right]$. Now because $a(\xi)>0$ and continuous in $[\alpha, \beta]$, it follows that $f^{\prime \prime}($.$) is continuous in compact$ sub-intervals, and hence in particular in $\left[r_{1}, r_{2}\right]$. Now for each $x$ in $[a, \beta]$

$$
\begin{aligned}
\frac{S(t) f(x)-f(x)}{t} & =\frac{1}{t} \int_{|\xi-x|>\varepsilon}[f(x)-f(\xi)] d P(t, \xi \mid x) \\
& +\frac{f^{\prime}(x)}{t} \int_{|\xi-x|<\varepsilon}(\xi-x) d P(t, \xi \mid x) \\
& +\frac{f^{\prime \prime}(x+\theta \varepsilon)}{2 t} \int_{|\xi-x|<\varepsilon}(\xi-x)^{2} d P(t, \xi x)
\end{aligned}
$$

where $0<|\theta|<1$.

In view of $L_{1}$, it follows that

$$
\operatorname{limit}_{t \rightarrow 0} \frac{T(t) f(x)-f(x)}{t}=a(x) f^{\prime \prime}(x)+b(x) f^{\prime}(x)
$$

and because of the asserted uniformity of the limits in $L_{1}$ and the conditions on $f($.$) , it is clear the limit in (2.6) is uniform in x$ in $[\alpha, \beta]$. 
Hence, for such $f($.$) it follows that$

$$
\Lambda f=C f .
$$

Moreover, for the same $f($.$) , note that C f$ again vanishes outside $\left[r_{1}, r_{2}\right]$. Also, $C f$ again belongs to the domain of $C$ and hence has first and second derivatives. Hence the argument above can be repeated to yield that

$$
\Lambda C f=C^{2} f
$$

and, of course

$$
C A f=C^{2} f
$$

or

$$
C A f=\Lambda C f .
$$

Denoting the semigroup generated by $C$ by $S(t)$ if follows readily that

$$
S(t) T(t) f=T(t) S(t) f
$$

and hence using the Dunford argument [See [7]]:

$$
S(t) f-T(t) f=\int_{0}^{t}(d / d \sigma) S(\sigma) T(\sigma) f=\int_{0}^{t} S(\sigma) T(\sigma)(\Lambda f-C f) d \sigma
$$

it follows that

$$
S(t)=T(t) f .
$$

It only remains to show that the set of such functions $f($.$) is dense in$ $C[\alpha, \beta]$. Now the class of functions in $C[\alpha, \beta]$ whose derivatives vanish outside compact subsets is dense in the domain of $C$. Because of the postulated twice differentiability of the coefficients $a(x)$ and $b(x)$, it follows that this class automatically belongs to the domain of $C^{2}$, proving the required denseness. It is quite probable the result holds without demanding differentiability of the functions $a(x)$ and $b(x)$.

This proves the theorem.

We note in passing that the conditions (2.3) do not imply uniqueness of solutions of the forward equation, as Hille [3] has shown. From our point of view, this lack of uniqueness is of no concern to us, thus avoiding problems associated with the duality between the backward and forward equations. In particular, Theorem 2.1 establishes that for $f($.$) in the domain of C$,

$$
E[f(x(t)) \mid x(0)]=u(t, x)
$$

is the unique solution of the Cauchy problem: 


$$
\frac{\partial u}{\partial t}=a(x) \frac{\partial^{2} u}{\partial x^{2}}+b(x) \frac{\partial u}{\partial x}
$$

with

$$
u(0, x)=f(x)
$$

As an example, consider the situation of Gaussian white-noise input to a nonlinear system, the input-output processes being related by [See Doob [2, p. 273] for the notation]

$$
d x(t)=\sqrt{2}\left(x(t)^{2}+1\right) d \zeta(t)
$$

$\zeta(t)$ being the real Gaussian additive process with

$$
E\left[|d \zeta(t)|^{2}\right]=d t
$$

so that the output process is Markoffian and we have for the limits in $L_{1}$ :

$$
\begin{aligned}
& a(x)=\left(x^{2}+1\right)^{2} \\
& b(x)=0 .
\end{aligned}
$$

These clearly satisfy all the required conditions of Theorem 2.1 and the predication function is the solution of the equation:

$$
\frac{\partial u}{\partial t}=\left(x^{2}+1\right)^{2} \frac{d^{2} u}{\partial x^{2}}
$$

subject to the initial condition

$$
u(0, x)=f(x)
$$

where it is assumed that

$$
\left(x^{2}+1\right) f^{\prime \prime}(x)
$$

belongs to $C[-\infty,+\infty]$.

In this particular case, we can obtain the solution in terms of orthogonal functions:

$$
u(t, x)=\sum_{0}^{\infty} a_{n} W_{n}(x) e^{-n(n+1) t}
$$

where

$$
\begin{aligned}
& a_{n}=\int_{-\infty}^{\infty} \frac{W_{n}(x)}{\left(x^{2}+1\right)^{2}} f(x) d x \\
& W_{n}(x)=\sqrt{\frac{2}{\pi}} \sqrt{\left(x^{2}+1\right)} \sin (n+1)\left(\frac{\pi}{2}-\arctan x\right) .
\end{aligned}
$$

[See Hille [5]] for this solution. The convergence of the series in (2.4) 
is uniform in $x$ in $[\alpha, \beta]$.

It may be noted that functions such as

$$
f(x)=x
$$

and

$$
f(x)=\exp i \lambda \cdot x
$$

are not in $C[-\infty,+\infty]$, so that we cannot obtain the prediction as the solution of the partial differential equation directly, in the sense in which we have stated that Cauchy problem. It may, however, be possible to consider a slightly different $B$-space such as the space of functions $f(x)$ continuous in $(-\infty, \infty)$ and such that

$$
\operatorname{limit}_{|x| \rightarrow \infty} f(x) \exp -|x|^{\rho}, \quad 0<\rho<1
$$

exist for some $\rho$, as Hille [6] does for the heat equation.

It should also be noted that in this example, the transition density kernel has the expansion

$$
p(t ; \xi \mid x)=\left(\xi^{2}+1\right)^{-2} \sum_{0}^{\infty} W_{n}(x) W_{n}(\xi) e^{-n(n+1) t} .
$$

As $t \rightarrow \infty$, we obtain the density

$$
p(\infty ; \xi \mid x)=2 / \pi\left(\xi^{2}+1\right)^{-2}
$$

and it should be noted that (2.9) for each $t$ is an orthogonal expansion with respect to this density. Also (2.10) corresponds to the (unique) stationary first order distribution with respect to which the process is ergodic. A sufficient condition for the existence of such an expansion (which automatically also yield the corresponding limiting density) due to Hille [5] is that in addition to (2.3) the following

$$
\int_{0}^{a} q^{\prime}(x) d x \int_{0}^{x} w(\xi) d \xi<+\infty \text { and } \int_{0}^{\beta} q^{\prime}(x) d x \int_{0}^{x} w(x) d x<\infty
$$

be also satisfied. In this case, the limiting density is simply

$$
\frac{q^{\prime}(x)}{q(\beta)-q(\alpha)} \text {. }
$$

All transition probabilities are absolutely continuous.

3. Markoff processes not of the diffusion type. We shall next consider the prediction problem not of the diffusion type, i.e., whose transition kernels do not satisfy conditions $L_{1}$, but rather an extended version of them, leading to elliptic partial differential equations. Thus, 
let the transition density kernels satisfy:

(i ) $2 p(t, \xi \mid y)-p(2 t, \xi \mid y) \geqq 0$

(ii) $\frac{1}{t^{2}} \int_{|y-\xi|>\varepsilon}[2 p(t, \xi \mid y)-p(2 t, \xi \mid y)] d \xi \rightarrow 0 \quad$ as $t \rightarrow 0+L_{2}$

(iii) $\frac{1}{t^{2}} \int_{|y-\xi|<\varepsilon}(\xi-y)[2 p(t, \xi \mid y)-p(2 t, \xi \mid y)] d \xi \rightarrow b(y)$

(iv) $\frac{1}{t^{2}} \int_{|y-\xi|<\varepsilon}(y-\xi)^{2}[2 p(t, \xi \mid y)-p(2 t, \xi \mid y)] d \xi \rightarrow 2 a(y)$.

Then the prediction function satisfies the elliptic partial differential equation:

$$
\frac{\partial^{2} u}{\partial t^{2}}+a(x) \frac{\partial^{2} u}{\partial x^{2}}+b(x) \frac{\partial u}{\partial x}=0 .
$$

As before, the main difficulty is in obtaining uniqueness of the solutions.

THEOREM 3.1. Suppose $a(y), b(y)$ are twice continously differentiable in $[\alpha, \beta]$ and $a(y)>0$ therein. Suppose further that the limits in $L_{1}$ hold uniformly in $y$ in each compact subinterval. Further, suppose that $a(\xi), b(\xi)$ satisfy (2.3). Then for each $f($.$) in the domain of C$,

$$
u(t, x)=E[f(x(t)) \mid x(0)=x]
$$

satisfies the partial differetial equation

$$
\frac{\partial^{2} u}{\partial t^{2}}+C u(\mathrm{t}, x)=0
$$

and is the only solution of it satisfying to the conditions:

$$
\begin{aligned}
& \text { (a) }\|u(t, .)-f(.)\| \rightarrow \text { as } t \rightarrow 0 \\
& \text { (b) }\|2 u(t, .)-u(2 t)\| \leqq\|f\| \\
& \text { (c) } \operatorname{Sup}_{t}\|u(t, .)\|<\infty .
\end{aligned}
$$

Conversely, suppose $a(\xi), b(\xi)$ are given such that they are continuous in $[\alpha, \beta]$ and $a(\xi)>0$ therein, and such that they satisfy (3.1). Then the Cauchy problem for (3.1) has a unique solution satisfying (a), (b) and (c) for each $f($.$) in the domain C$, the solution being given by

$$
u(t, x)=\int_{\alpha}^{\beta} f(\xi) p(t, \xi \mid x) d \xi
$$

where the $p(t, \xi \mid x)$ are Markoff transition densities satisfying $L_{2}$, the limits existing uniformly in $y$ in compact sub-intervals.

Proof. For a proof of the converse part [see [6]]. Since $a(),. b($. 
satisfy these conditions in the forward part as well, let us denote the corresponding semigroup by $T(t)$ with generator $B$. Then we know that

$$
B^{2}=-C \text {. }
$$

For each $f($.$) in C[\alpha, \beta]$ let us next let

$$
u(t, x)=E[f(x(t)) \mid x(0)=x] .
$$

Then the conditions $L_{2}$ on the transition kernels imply that $u(t, x)$ satisfies (a), (b) and (c), and moreover setting

$$
u(t, x)=S(t) f(x)
$$

$S(t)$ is a strongly continuous semigroup over $C[\alpha, \beta]$. Let us denote its generator by $\Lambda$. We have now to show that

$$
\Lambda^{2}=B^{2}=-C \text {. }
$$

For this, let $f($.$) belong to C[\alpha, \beta]$ and let $f^{\prime}($.$) vanish outside a compact$ sub-interval $\left[r_{1}, r_{2}\right]$. Then as in the proof of Theorem 2.1, we shall first show that $f($.$) belongs also to the domain of \Lambda^{2}$ and that

$$
\Lambda^{2} f=B^{2} f \text {. }
$$

For this we note that

$$
\begin{aligned}
& \frac{T(2 t)+I-2 T(t)}{t^{2}} f(x) \\
& =\frac{1}{t^{2}} \int_{-\infty}^{\infty}(f(\xi)-f(x))[p(2 t, \xi \mid x)-2 p(t, \xi \mid x)] d \xi
\end{aligned}
$$

and as before, as $t \rightarrow 0$, by virtue of $L_{2}$ this goes to

$$
-a(x) f^{\prime \prime}(x)-b(x) f^{\prime}(x)
$$

and the rest of the arguments go over similarly. Also we readily obtain that:

$$
\Lambda^{2} B^{2} f=B^{2} \Lambda^{2} f .
$$

This is enough to imply that

$$
T(t) f=S(t) f
$$

and the differentiability properties of $a(x)$ and $b(x)$ again imply that such functions $f($.$) are dense in the domain of C$ and hence (3.2) follows. This concludes the proof.

The simplest example of a process with transition kernels satisfying the conditions $L_{2}$ is the Cauchy additive process, with the independent increments having a Cauchy distribution: 


$$
E[\exp i s(\zeta(t+\Delta)-\zeta(t))]=\exp -|s| \Delta .
$$

More generally, such a process arises as the output of a first-order system whose imput is the Cauchy additive process:

$$
d x(t)=b(x(t) d t+a(x(t)) d \zeta(t)
$$

in the notation of Doob (loc. cit.), $\zeta(t)$ being the input Cauchy additive process ('non-Gaussian white noise'). Now

$$
x(t+\Delta)-x(t)=b[x(t)] \Delta+a[x(t)][\zeta(t+\Delta)-\zeta(t)]
$$

where the right-side, for given $x(t)$ is specified in terms of $\zeta(t)$ whose statistics are known. The limits required in $L_{2}$ are then established by direct calculation. In the case of (i), we may note that we need only prove it for small $t$, since the semigroup property will then imply it for all values of $t$. We omit the details of these calculations. The differential equation is:

$$
\frac{\partial^{2} u}{\partial t^{2}}+\frac{a(x)^{2}}{2} \frac{\partial^{2} u}{\partial x^{2}}+b(x) \frac{\partial u}{\partial x}=0 .
$$

As an example we may consider the case where: $a($.$) and b($.$) are$ constants:

$$
\begin{aligned}
& a(x)=\sqrt{2} \\
& b(x)=-2 x .
\end{aligned}
$$

The differential equation then is:

$$
\frac{\partial^{2} u}{\partial t^{2}}=-2 x \frac{\partial u}{\partial x}+\frac{\partial^{2} u}{\partial x^{2}} .
$$

The (unique) solution of this is the prescribed type for each initial function $f($.$) can be expanded in Hermite polynomials [See [6] for a general$ proof]

$$
u(t, x)=\sum_{0}^{\infty} a_{n} H_{n}(x) \exp -\sqrt{2 n} t
$$

where the $H_{n}($.$) are the Hermite polynomials orthogonal with respect$ to the Gaussian density:

$$
(1 / \sqrt{\pi}) \exp -x^{2}
$$

and

$$
a_{n}=\frac{1}{\sqrt{\pi}} \int_{-\infty}^{\infty} f(x) H_{n}(x) \exp -x^{2} d x .
$$

The series in (3.3) converges to the solution function uniformly in com- 
pact subsets of $(-\infty,+\infty)$. The transition kernel density $p(t, \xi \mid x)$ is given by

$$
p(t, \xi \mid x)=\frac{1}{2 \pi} \int_{-\infty}^{\infty} \frac{t}{\sqrt{\sigma^{2}(1-\exp -2 \sigma)}} \exp \left[\frac{-[\xi-x \exp -2 \sigma]^{2}}{(1-\exp -2 \sigma)}-\frac{t^{2}}{4 \sigma}\right] \mathrm{d} \sigma
$$

as follows again from the theory in [6]. Alternately, it has the expansion:

$$
p(t, \xi \mid x)=\sum_{0}^{\infty} H_{n}(x) H_{n}(\xi) \exp -\xi^{2}-\sqrt{2 n} t .
$$

As $t \rightarrow \infty$, the limiting density is: Gaussian:

$$
p(\xi)=\frac{1}{\sqrt{\pi}} \exp -\xi^{2}
$$

with respect to which (as first-order density) the process becomes strictly stationary. It may be shown that the limiting density is again always given by

$$
p^{\prime}(x) /(q(\beta)-q(\alpha))
$$

and is thus completely determined by the system, that is by $a($.$) and$ $b($.$) only. The expansion (3.3) is, of course, in terms of functions$ orthogonal with respect to this density. Thus, taking the example treated in $\S 2$, with

$$
\begin{aligned}
& a(x)=\left(x^{2}+1\right) \sqrt{2} \\
& b(x)=0
\end{aligned}
$$

yielding the differential equation:

$$
\frac{\partial^{2} u}{\partial t^{2}}+\left(x^{2}+1\right)^{2} \frac{\partial^{2} u}{2 x^{2}}=0
$$

we have the expansion:

$$
u(t, x)=\sum_{0}^{\infty} a_{n} W_{n}(x) \exp -\sqrt{n(n-1)} t
$$

with $W_{n}(x)$ and $a_{n}$ as in (2.3). As before, a sufficient condition for the existence of such expansion, is that (2.3) and (2.11) be satisfied. However, this is not necessary as the previous example (3.3) shows.

Extensions. A generalization of the type of process treated in $\S 3$ is got by replacing the kernels in $L_{2}$ by

$$
\frac{1}{t^{n}}\left[\sum_{1}^{n}\left(\begin{array}{l}
n \\
r
\end{array}\right)(-1)^{r-1} p(r t, \xi \mid x)\right]
$$


$\left(\begin{array}{l}n \\ r\end{array}\right)$ being the Binomial coefficients, leading to the equations

$$
\frac{\partial^{n} u}{\partial t^{n}}=(-1)^{n+1}\left[a(x) \frac{\partial^{2} u}{\partial x^{2}}+b(x) \frac{\partial u}{\partial x}\right] .
$$

However, we have been unable as yet to establish the conditions for uniqueness of the solutions.

We have only so far considered first-order Markoff processes. The extension to higher order processes is similar in principle although it entails partial differential equations in several space variables [see [8] for example], and the results on the related Cauchy problems are still incomplete to a large degree.

\section{REFERENCES}

1. A. V. Balakrishnan, On a class of elliptic equations in the theory of Markoff processes, Journal of Mathematics and Mechanics, Vol. 10, No. 4, July 1961.

2. J. L. Doob, Stochastic Processes, John Wiley and Sons, New York, 1954.

3. W. Feller, Zur theorie der stochastischen prozesse (existence und eindeutigkeitssatze), Math. Annalen, 113 (1936), 113-160.

4. On positivity preserving semigroups of transformations on $C\left[r_{1}, r_{2}\right]$, Annales Polonaize, (1952), 85-94.

5. E. Hille, The abstract Cauchy problem and Cauchy's problem for parabolic equations, Journal d'Analyse Mathematique, (1953/54), 81-196.

6. 15 (1956), 102-118.

, Quelque remarques sur l'equation de la chaleur, Rend. Mat. e Appe. (5),

7. E. Hille and R. S. Phillips, Functional analysis and semigroups, A.M.S. Colloquium Publications, 31 (1958).

8. N. C. Wang and G. E. Uhlenbeck, On the theory of Browian motion II, Reviews of Modern Physics, 17 (1945) 323-342. 


\section{PACIFIC JOURNAL OF MATHEMATICS}

\section{EDITORS}

\author{
RaLPh S. Phillips \\ Stanford University \\ Stanford, California \\ F. H. BRowNELL \\ University of Washington \\ Seattle 5 , Washington
}

A. L. Whiteman

University of Southern California

Los Angeles 7, California

L. J. Paige

University of California

Los Angeles 24, California

\author{
E. F. BECKENBACH \\ T. M. CHERRY
}

\author{
ASSOCIATE EDITORS

$\begin{array}{lll}\text { D. DERRY } & \text { H. L. ROYDEN } & \text { E. G. STRAUS } \\ \text { M. OHTSUKA } & \text { E. SPANIER } & \text { F. WOLF }\end{array}$

\section{SUPPORTING INSTITUTIONS}

\author{
UNIVERSITY OF BRITISH COLUMBIA \\ CALIFORNIA INSTITUTE OF TECHNOLOGY \\ UNIVERSITY OF CALIFORNIA \\ MONTANA STATE UNIVERSITY \\ UNIVERSITY OF NEVADA \\ NEW MEXICO STATE UNIVERSITY \\ OREGON STATE COLLEGE \\ UNIVERSITY OF OREGON \\ OSAKA UNIVERSITY \\ UNIVERSITY OF SOUTHERN CALIFORNIA
}

\author{
STANFORD UNIVERSITY \\ UNIVERSITY OF TOKYO \\ UNIVERSITY OF UTAH \\ WASHINGTON STATE COLLEGE \\ UNIVERSITY OF WASHINGTON \\ AMERICAN MATHEMATICAL SOCIETY \\ CALIFORNIA RESEARCH CORPORATION \\ HUGHES AIRCRAFT COMPANY \\ SPACE TECHNOLOGY LABORATORIES \\ NAVAL ORDNANCE TEST STATION
}

Mathematical papers intended for publication in the Pacific Journal of Mathematics should be typewritten (double spaced), and the author should keep a complete copy. Manuscripts may be sent to any one of the four editors. All other communications to the editors should be addressed to the managing editor, L. J. Paige at the University of California, Los Angeles 24, California.

50 reprints per author of each article are furnished free of charge; additional copies may be obtained at cost in multiples of 50 .

The Pacific Journal of Mathematics is published quarterly, in March, June, September, and December. The price per volume (4 numbers) is $\$ 12.00$; single issues, $\$ 3.50$. Back numbers are available. Special price to individual faculty members of supporting institutions and to individual members of the American Mathematical Society: $\$ 4.00$ per volume; single issues, $\$ 1.25$.

Subscriptions, orders for back numbers, and changes of address should be sent to Pacific Journal of Mathematics, 103 Highland Boulevard, Berkeley 8, California.

Printed at Kokusai Bunken Insatsusha (International Academic Printing Co., Ltd.), No. 6, 2-chome, Fujimi-cho, Chiyoda-ku, Tokyo, Japan.

\section{PUBLISHED BY PACIFIC JOURNAL OF MATHEMATICS, A NON-PROFIT CORPORATION}

The Supporting Institutions listed above contribute to the cost of publication of this Journal, but they are not owners or publishers and have no responsibility for its content or policies.

Reprinted 1966 in the United States of America 


\section{Pacific Journal of Mathematics}

\section{Vol. 11, No. 4}

A. V. Balakrishnan, Prediction theory for Markoff processes . . . . . . . . . . 1171

Dallas O. Banks, Upper bounds for the eigenvalues of some vibrating systems . . . . 1183

A. Białynicki-Birula, On the field of rational functions of algebraic groups ...... 1205

Thomas Andrew Brown, Simple paths on convex polyhedra .............. 1211

L. Carlitz, Some congruences for the Bell polynomials . . . . . . . . . . . . 1215

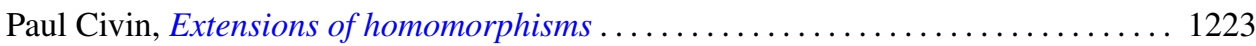

Paul Joseph Cohen and Milton Lees, Asymptotic decay of solutions of differential

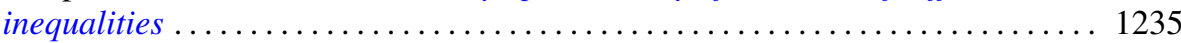

István Fáry, Self-intersection of a sphere on a complex quadric . . . . . . . . . . 1251

Walter Feit and John Griggs Thompson, Groups which have a faithful representation



William James Firey, Mean cross-section measures of harmonic means of convex

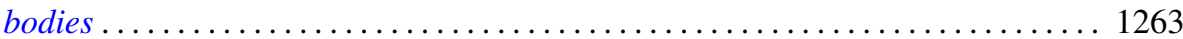

Avner Friedman, The wave equation for differential forms . . . . . . . . . . 1267

Bernard Russel Gelbaum and Jesus Gil De Lamadrid, Bases of tensor products of

Banach spaces ................................... 1281

Ronald Kay Getoor, Infinitely divisible probabilities on the hyperbolic plane . . . . 1287

Basil Gordon, Sequences in groups with distinct partial products . . . . . . . . . . . . 1309

Magnus R. Hestenes, Relative self-adjoint operators in Hilbert space . . . . . . . . . 1315

Fu Cheng Hsiang, On a theorem of Fejér ......................... 1359

John McCormick Irwin and Elbert A. Walker, On N-high subgroups of Abelian

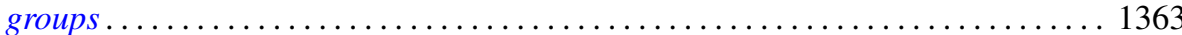

John McCormick Irwin, High subgroups of Abelian torsion groups . . . . . . . . . 1375

R. E. Johnson, Quotient rings of rings with zero singular ideal . . . . . . . . . . . 1385

David G. Kendall and John Leonard Mott, The asymptotic distribution of the time-to-escape for comets strongly bound to the solar system ...

Kurt Kreith, The spectrum of singular self-adjoint elliptic operators ....

Lionello Lombardi, The semicontinuity of the most general integral of the calculus of variations in non-parametric form ................................

Albert W. Marshall and Ingram Olkin, Game theoretic proof that Chebyshev inequalities are sharp

Wallace Smith Martindale, III, Primitive algebras with involution . . William H. Mills, Decomposition of holomorphs ..............

James Donald Monk, On the representation theory for cylindric algebras . . . . . . 1447

Shu-Teh Chen Moy, A note on generalizations of Shannon-McMillan theorem . . . . 1459

Donald Earl Myers, An imbedding space for Schwartz distributions . .

John R. Myhill, Category methods in recursion theory .........

Paul Adrian Nickel, On extremal properties for annular radial and circular slit mappings of bordered Riemann surfaces

Edward Scott O'Keefe, Primal clusters of two-element algebras . .

Nelson Onuchic, Applications of the topological method of Wazewski to certain

problems of asymptotic behavior in ordinary differential equations ...

Peter Perkins, A theorem on regular matrices................

Clinton M. Petty, Centroid surfaces .... 